\title{
Aerodynamic shape optimization of civil structures: a CFD-enabled kriging-based approach
}

\author{
Enrica Bernardini ${ }^{\mathrm{a}}$, Seymour M.J. Spence ${ }^{\mathrm{b}, 1, *}$, Daniel Wei ${ }^{\mathrm{a}}$, Ahsan Kareem ${ }^{\mathrm{c}}$ \\ ${ }^{a}$ Formerly at NatHaz Modeling Laboratory, Department of Civil and Environmental \\ Engineering and Earth Sciences, University of Notre Dame, Notre Dame, IN 46556, USA \\ ${ }^{b}$ Department of Civil and Environmental Engineering, University of Michigan, Ann \\ Arbor, MI 48103, USA \\ ${ }^{c}$ NatHaz Modeling Laboratory, Department of Civil and Environmental Engineering and \\ Earth Sciences, University of Notre Dame, Notre Dame, IN 46556, USA
}

\section{Abstract}

In the case of mega-structures such as tall buildings and long-span bridges, the mitigation of the intensity of the wind excitation through aerodynamic tailoring of the external shape can be fundamental for meeting the performance goals. The search for the best performing shape through an automatic CFD-enabled optimization methodology is potentially less expensive, less time-consuming and more thorough than the common trial-and-error approach based on wind tunnel test results, therefore very attractive. This paper investigates the possibility of carrying out the multi-objective aerodynamic shape optimization of civil structures through an approach in which evolutionary algorithms are used in synergy with ordinary kriging surrogates. A specifically developed strategy is adopted to update the kriging models

\footnotetext{
${ }^{*}$ Corresponding author

Email addresses: enricabernardini14@gmail.com (Enrica Bernardini), smjs@umich.edu (Seymour M.J. Spence), lakeat@gmail.com (Daniel Wei), kareem@nd.edu (Ahsan Kareem)

${ }^{1}$ Tel. +1 734764 8419, Fax +1 7347644292
}

Preprint submitted to Elsevier

March 16, 2015

(C) 2015. This manuscript version is made available under the Elsevier user license http://www.elsevier.com/open-access/userlicense/1.0/ 
making efficient use of additional CFD runs. Shell scripting, parallelized computations and mesh morphing algorithms are exploited for enhancing the framework's efficiency and consistency. As a case study, the optimization of the shape of a tall building cross-section in terms of both the lift and the drag coefficient is considered.

Keywords: Aerodynamic shape optimization, Kriging, CFD, Tall buildings

\section{1. Introduction}

2

3

4

5

6

7

8

In recent years, the number of high-rise buildings and long-span bridges constructed all over the world has exponentially increased, with a trend towards taller, or longer, more slender and lighter structures. A consequence of this trend is a heightened sensitivity to the action of wind, which makes the satisfaction of the required structural performance in terms of survivability, serviceability and habitability more challenging for the designers, even when the best possible choice of structural system is made. The mitigation of the intensity of the wind excitation through aerodynamic tailoring of the external shape can therefore be fundamental for meeting the performance goals, potentially eliminating the necessity of more expensive alternative solutions, which typically involve the use of auxiliary motion control devices. The advantages that can be had through a suitable choice of the shape are indeed well known. In the case of high-rise buildings, it has been observed that modifications of the cross-section such as chamfering and recession of corners can significantly reduce the alongwind and acrosswind response (Kareem et al., 1999); also, variations along the vertical axis, such as variation of the crosssection, tapering, or the introduction of helical profiles or setbacks (Kim 
et al., 2011; Tanaka et al., 2012, e.g.) are known to have beneficial effects.

For bridges, aerodynamically tailored slotted box sections are often adopted in order to minimize the aerodynamic loads and shift flutter to higher wind speeds; also, edge treatments such as fairings and deck extensions are used to improve the aerodynamic behavior, even in the case of retrofitting (Kareem et al., 2013a).

To take advantage of the aerodynamic shape tailoring in the case of extreme structures particularly affected by the wind action, what is commonly done is to consider, in the preliminary phases of the design process, more than one configuration in order to identify the less aerodynamically demanding (Abdelrazaq et al., 2004; Baker et al., 2007; Xie, 2014, e.g.). Typically, wind tunnel tests are used to characterize the aerodynamic behavior of the candidate shapes, selected a priori based on experience, therefore the number of configurations that can be considered is limited by the significant resources and time necessary to execute each test. As a consequence, a vast portion of the search space remains unexplored, and more conventional configurations are favored over innovative solutions.

The possibility of taking advantage of computational fluid dynamics (CFD) simulations for the assessment of the aerodynamic performance while using optimization algorithms to find the best aerodynamic shape is therefore very attractive as it would allow not only to rigorously and thoroughly investigate the search domain, but to do so automatically, also in principle eliminating the necessity of costly wind tunnel experiments. This idea is not new in the fields of mechanical, automotive and aerospace engineering, where CFDbased aerodynamic shape optimization (ASO) has been applied to discover 
optimal geometric configurations for vehicles, aircraft bodies and wings, compressor blades, laminar and turbulent flow diffusers etc. (Madsen et al., 2000; Mohammadi and Pironneau, 2001; Thévenin and Janiga, 2008, e.g.). In the 47 field of civil engineering, however, ASO is only recently gaining interest (Kareem et al., 2014, 2013b; Spence et al., 2013). This is probably due, on one

hand, to the fact that the traditional trial-and-error approach to the design has so far been proved sufficient for most structures. Also, the intrinsic link between the aesthetics of the structure and the structural form tends to raise skepticism regarding the applicability of ASO, which, suggesting that the shape of a civil structure can be found as the solution of a purely mathematical problem, appears incompatible with architectural considerations. On the other hand, the implementation of ASO which relies on CFD simulations is intrinsically difficult for civil structures, due to the bluff nature of the bodies, the turbulent and separated nature of the flow field and the presence of the boundary layer in which the structures are immersed, the high values assumed by the Reynolds number, the multi-objective nature of the design problem.

This paper investigates the possibility of carrying out the multi-objective CFD-based ASO of civil structures through a surrogate-based approach. The basic idea is to use a limited number of CFD simulations to build surrogate models which, being far less expensive to evaluate, can be explored through evolutionary algorithms to find the optimal solutions. In particular, in this work the possibility of using ordinary kriging for the construction of the surrogates is investigated. A specifically developed strategy is adopted to update the kriging models making efficient use of additional CFD runs while 
taking advantage of shell scripting and parallelized computations. Suitably defined constraints are used to define the range of admissible shape variations. As a case study, the optimization of the shape of a tall building cross-section in terms of both the standard deviation of the lift coefficient and the mean drag coefficient is considered, where URANS (unsteady Reynolds-averaged Navier-Stokes) CFD simulations are adopted for modeling the turbulent flow and therefore also for calibrating the kriging models.

\section{Problem statement}

The idea behind shape optimization is to allow the boundary of a body to be modified while satisfying certain constraints in order to attain the best possible performance. After having described the geometry in terms of a set of $n$ parameters collected in the vector of the design variables $\mathbf{q}$, the shape optimization problem can be posed as follows:

$$
\text { Find } \mathbf{q}=\left(q_{1}, q_{2}, \ldots, q_{n}\right)
$$

$$
\text { to minimize } \mathbf{G}(\mathbf{q})
$$

subject to:

$$
\begin{aligned}
& C_{r}(\mathbf{q})=0 \quad r=1, \ldots, R \\
& D_{s}(\mathbf{q}) \leq 0 \quad s=1, \ldots, S
\end{aligned}
$$

where $\mathbf{G}(\mathbf{q})=\left[G_{1}(\mathbf{q}), \ldots, G_{N}(\mathbf{q})\right]$ is a vector collecting the $N$ objective functions, while Eqs. 3 and 4 represent $R$ equality constraints, and $S$ inequality constraints, respectively, imposed on the design variables. The set of feasible solutions is given by all the vectors $\mathbf{q}$ that satisfy the constraints of Eqs. 3 
and 4. A feasible vector $\mathbf{q}^{*}$ is a Pareto-optimal solution of the multi-objective optimization problem if it is not possible to find another feasible solution for which an improvement in one objective does not correspond to a degradation in one or more of the others. In mathematical terms, a vector $\mathbf{q}^{*}$ is a Pareto-optimal solution if it is feasible and there exists no other feasible $\mathbf{q}$ such that 1) $G_{j}(\mathbf{q}) \leq G_{j}\left(\mathbf{q}^{*}\right)$ for all $j$ and 2) $G_{i}(\mathbf{q})<G_{i}\left(\mathbf{q}^{*}\right)$ for at least one $i$. In general, the various objective functions are competing, and more than one Pareto-optimal solution exists. From the viewpoint of the mathematical problem formulation these solutions are all equally good. It is therefore for the decision-makers to choose, based on additional considerations, the "best" trade-off solution among the ones belonging to the Pareto-optimal set, and for the optimization algorithm to find Pareto-optimal solutions as diverse as possible in the objective function space. This in particular is not trivial due to the high non-linearity often characterizing the mapping between design variables and objective functions (Forrester et al., 2008).

Being here of interest the study of the aerodynamic behavior of the body, one or more of the objective functions and/or of the constraint functions of the optimization problem will be in terms of aerodynamic quantities that have to be evaluated through CFD simulations. The significant computational effort required to carry out even the simplest CFD simulations represents the crucial difficulty of ASO, as it makes unfeasible the straightforward use of classic optimization strategies which need numerous function calls. The following section presents the strategy used here to tackle this challenge. 


\section{Solution strategy}

\subsection{Optimization algorithms}

The strategy proposed in this work for solving the ASO problem entails the use of evolutionary algorithms (EAs). These algorithms mimic the evolution of species and the survival of the fittest by considering a population of individuals, each one characterized by a genome, evolving from generation to generation through biologically inspired mechanisms such as crossover, mutation and selection. The genome of an individual is a set of values assumed by the design variables while the fitness of the individual is described by the values of the objective functions. EAs have often been preferred to gradientbased techniques in the case of ASO problems due to several advantages that make them successful in a variety of applications. In particular, their robustness allows them to handle very large design spaces that are characterized by irregular landscapes, without getting trapped into local optima; they are also easy to parallelize (the performances of the various individuals of the population are independent of each other), easy to hybridize with alternative methods and simple to program. Even more importantly in the case here considered, EAs are particularly suitable for multiobjective optimization problems because they are able to find the entire range of Pareto-optimal solutions while keeping them as diverse as possible (Arias-Montaño et al., 2012; Deb, 2001), while gradient-based methods can handle multiobjective optimization problems only by combining the various objectives into one, typically using a weighting method, which results to be dependent on the chosen weighting coefficients.

While the advantages of using EAs for the problem of ASO are clear, 
these algorithms require by nature an extremely high number of function calls (which depends on the size of the population and on the number of generations required to converge, and can easily reach the order of thousands). The use of EAs for problems where the objective or constraint functions are evaluated through CFD simulations is therefore not feasible unless a surrogate-based approach is adopted.

\subsection{Surrogate modeling}

\subsubsection{Overview}

Surrogate modeling consists in replacing a function $f(\mathbf{x})$ which is expensive to evaluate with an inexpensive surrogate (emulator, or meta-model) $\hat{f}(\mathbf{x})$, which has the same input and output space of the original function. In surrogate-based optimization, the surrogate replaces one or more of the objective functions (and/or of the constraint functions), and the search for the optimum is therefore carried out on the surrogate model, which has to be previously constructed based on a limited, but carefully chosen, number of runs of the original function. This set of sample points $\left(\overline{\mathbf{x}}^{(1)}, \ldots, \overline{\mathbf{x}}^{(h)}\right)$ is termed sampling plan. The identification of the optimum through the use of the surrogate(s) is followed, in general, by a model validation phase, in which the predictive capabilities of the surrogate model are tested, and then by an improvement phase, where the surrogate is updated in order to enhance the quality of the approximation.

\subsubsection{The kriging surrogate}

Different surrogate models have been adopted in the field of design optimization, such as polynomial regression, radial basis functions, neural net- 
works, support vector regression (Ahmed and Qin, 2009). In this work, the extremely popular ordinary kriging has been chosen (Sacks et al., 1989), because of its ability to represent a variety of different typologies of functions and of providing, in addition to the prediction, an estimate of the prediction error that will be exploited in the model improvement phase.

The prediction of the kriging surrogate is based on the assumption that the function that has to be modeled can be seen as a realization of a Gaussian stochastic process, i.e. the deviation of the value assumed by the function at a point from its mean is interpreted as a random process, more precisely as a stationary covariance process (with zero mean) (Kleijnen, 2009). Given the set of the $h$ observed data $\overline{\mathbf{y}}=\left[f\left(\overline{\mathbf{x}}^{(1)}\right), \ldots, f\left(\overline{\mathbf{x}}^{(h)}\right)\right]^{\prime}$, the kriging prediction at the location $\mathbf{x}=\left(x_{1}, \ldots, x_{n}\right)^{\prime}$ is expressed as:

$$
\hat{f}(\mathbf{x})=\hat{\mu}+\boldsymbol{\psi}^{\prime} \mathbf{R}^{-1}(\overline{\mathbf{y}}-\mathbf{1} \hat{\mu})
$$

where $\hat{\mu}$ is the maximum likelihood estimator of the mean of the random field, $\boldsymbol{\psi}$ is an $h \times 1$ vector of functions that can be interpreted as basis functions (Forrester et al., 2008), while the term $\mathbf{R}^{-1}(\overline{\mathbf{y}}-\mathbf{1} \hat{\mu})$ represents the weights assigned to the basis functions ( $\mathbf{1}$ is the $h \times 1$ unit vector). The basis functions and the terms of the $h \times h$ matrix $\mathbf{R}$ result from the assumed correlation function, which, according to a popular choice, is given by:

$$
\operatorname{Corr}\left[f\left(\mathbf{x}^{(l)}\right), f\left(\mathbf{x}^{(m)}\right)\right]=\exp \left(\sum_{k=1}^{n} \theta_{k}\left\|x_{k}^{(l)}-x_{k}^{(m)}\right\|^{p_{k}}\right)
$$

where $\theta_{k}$ is the $k$ th component of a vector of parameters $\boldsymbol{\theta}$ and $p_{k}$ is the $k$ th component of a vector of parameters $\mathbf{p}$. In particular, the $i$ th function of the vector $\boldsymbol{\psi}$ is given by:

$$
\psi_{i}(\mathbf{x})=\operatorname{Corr}\left[f\left(\overline{\mathbf{x}}^{(i)}\right), f(\mathbf{x})\right]
$$


while the $(i, j)$ th element of $\mathbf{R}$ represents the correlation between the $i$ th and the $j$ th sample points and is therefore given by:

$$
R_{i, j}=\operatorname{Corr}\left[f\left(\overline{\mathbf{x}}^{(i)}\right), f\left(\overline{\mathbf{x}}^{(j)}\right)\right]
$$

The $2 n$ model parameters $\boldsymbol{\theta}$ and $\mathbf{p}$ are selected in order to maximize the likelihood of the set of the $h$ observed data $\overline{\mathbf{y}}$. This is practically done by maximizing the so-called concentrated log-likelihood function $(C L L)$, given by (Jones, 2001):

$$
C L L=-\frac{h}{2} \ln \left(\hat{\sigma}^{2}\right)-\frac{1}{2} \ln (|\mathbf{R}|)
$$

where $\hat{\sigma}^{2}$ is the maximum likelihood estimator of the variance of the random field, given by:

$$
\hat{\sigma}^{2}=\frac{1}{h}(\overline{\mathbf{y}}-\mathbf{1} \hat{\mu})^{\prime} \mathbf{R}^{-1}(\overline{\mathbf{y}}-\mathbf{1} \hat{\mu})
$$

with

$$
\hat{\mu}=\frac{\mathbf{1}^{\prime} \mathbf{R}^{-1} \overline{\mathbf{y}}}{\mathbf{1}^{\prime} \mathbf{R}^{-1} \mathbf{1}}
$$

191 In practice, to simplify the maximization of $C L L$, the values of the parameters $\mathbf{p}$ are often chosen. In this work, $p_{k}=2$ will be assumed for $k=1, \ldots, n$, which gives the so-called Gaussian correlation function (Eq. 6). 195 et al., 1989):

$$
\hat{s}^{2}=\hat{\sigma}^{2}\left[1-\boldsymbol{\psi}^{\prime} \mathbf{R}^{-1} \boldsymbol{\psi}+\frac{\left(1-\boldsymbol{\psi}^{\prime} \mathbf{R}^{-1} \boldsymbol{\psi}\right)^{2}}{\mathbf{1}^{\prime} \mathbf{R}^{-1} \mathbf{1}}\right]
$$

${ }_{196}$ The third term inside the square brackets is small and it is often neglected. ${ }_{197}$ The mean squared error $\hat{s}^{2}$ will be useful in the implementation of the im198 provement strategy that will be illustrated in Section 3.3.2. 


\subsubsection{Design of experiments}

The choice of the sampling points to calibrate the kriging surrogate is obviously of fundamental importance for its accuracy. In general, the idea is that the bias error of the surrogate can be reduced for sampling plans that more evenly fill the space, given the total number of points. One of the most popular choices for creating a sampling plan is the use of optimal Latin hypercube designs. A Latin hypercube (Mackay et al., 1979) is a sampling plan for which the projections of all the points on each axis result in a uniform distribution over the entire range of variation of the axis variable. Optimal Latin hypercubes are particular Latin hypercubes characterized by optimality from the viewpoint of space-fillingness (a Latin hypercube does not necessarily have good space-filling properties). In this paper, optimal Latin hypercube sampling with the maximin criterion given by Morris and Mitchell (1995) is used to generate the sampling plan.

It should be observed that the function evaluations for the points of the sampling plan, being independent of each other, can be carried out in parallel. This means that it is convenient to adjust the size of the sampling plan to the maximum number of possible parallel computations. If $N_{p}$ is the maximum number of possible parallel computations, the size of the sampling plan should be chosen in order to be equal to $N_{p}$ or, if this is deemed not sufficient, a multiple of $N_{p}$ (i.e. batches of $N_{p}$ analyses carried out sequentially).

\subsection{Surrogate validation and improvement}

The surrogate validation and improvement is a very delicate phase that considerably influences the effectiveness and efficiency of the surrogate-based optimization procedure. In the following, considerations on the definition of 
updating strategies are reported, followed by the description of the strategy that will be applied to the case study presented in this work.

\subsubsection{Considerations on updating strategies}

Once the surrogate-based optimization has provided the set of Paretooptimal solutions, the more intuitive and simpler way of testing the validity of the surrogate is running additional analyses of the expensive function on representative points of the Pareto set and comparing the outcomes to the predictions. If the comparison is not satisfactory, an update of the surrogate is necessary, and this can be done by simply re-calibrating the surrogate to the set of the original sampling points plus the additional sampling points. This procedure results in a local improvement of the surrogate that can be very effective. If however the initial global accuracy is poor, it can lead to the identification of a local minimum (Queipo et al., 2005; Sóbester et al., 2004).

A more sophisticated way of identifying the locations of the design space where new sampling points should be added to obtain the best benefits in terms of the surrogate quality is based on exploiting the stochastic features of kriging. In particular, updating strategies based on the expected improvement have been proposed (Jones et al., 1998; Schonlau et al., 1998) and have been demonstrated to be very effective (Forrester et al., 2008), because they tend to improve the global quality of the approximation. The idea is to add points at the locations where the expectation of the improvement in the objective function given by the added points is higher. Note that the improvement, however defined, is a random variable because the value of the function $f$ at a point $\mathbf{x}$ is uncertain. 
In the context of single objective optimization, if $G=f$ is the objective function to be minimized, denoting by $G_{\min }$ the best objective value from the sample evaluated so far, the improvement can be defined as:

$$
I(\mathbf{x})=\max _{\mathbf{x}}\left[G_{\min }-Y, 0\right]
$$

where $Y$ represents the random variable modeling the uncertainty about the value of the function $G$ at $\mathbf{x}$, and therefore is normally distributed with mean $\hat{\mu}$ (Eq. 11) and variance $\hat{s}^{2}$ (Eq. 12). The expected improvement is obtained by taking the expected value of $I$ :

$$
E[I(\mathbf{x})]=\int_{-\infty}^{G_{\min }}\left(G_{\min }-y\right) \phi(y) d y
$$

where $\phi$ is the probability density function of $Y$ (Jeong et al., 2006).

In the case of more than one objective approximated by a surrogate, the application of the expected improvement criterion is not as straightforward, because the presence of more than one surrogate implies the necessity of a redefinition of the concept of improvement. To overcome this difficulty, Jeong et al. (2006) proposed to evaluate the expected improvement of each objective function and find the new sample points as optima of a multi-objective optimization problem, i.e. as the Pareto-optimal solutions that maximize the expected improvement of all the objectives. Shimoyama et al. (2013) define the improvement based on a hypervolume indicator, which gives a measure of the quality of a set of solutions. In the work of Keane (2006), given a Pareto set, a new design point is considered to give an improvement if it becomes member of the Pareto set (i.e. the Pareto set is augmented) or if it comes to substitute one of the points of the Pareto set (i.e. it dominates at least one of its members). Either one of these possibilities, as well as a combination 
of them, can therefore be taken as metric. Having defined the improvement, its expected value is calculated by considering uncorrelated kriging models, built on the set of training points for the various objective functions, and defining an $N$-dimensional Gaussian probability density function ( $N$ is the number of objective functions). The probability density function will depend on the statistics of the prediction given by the $N$ kriging models $\left(\hat{\mu}_{1}, \hat{s}_{1}^{2}, \ldots\right.$, $\left.\hat{\mu}_{N}, \hat{s}_{N}^{2}\right)$ and will allow the estimation of the expected improvement. For the case $N=2$, in Keane (2006) a geometric interpretation is provided: the improvement metric is given by the first moment of the joint probability density function integral over the area where improvements occur, about the Pareto front (the analogy with the case $N=1$ is immediate by observing Eq. 14). The geometrical interpretation is lost for cases with higher $N$; nevertheless the metric remains valid and expressible in closed form. In the following, the expected improvement criterion given by Keane (2006) with improvement considered when the added point dominates (and not augments) the Pareto set will be adopted in the definition of an updating strategy.

Another interesting observation that will be kept in mind when defining the updating strategy adopted in this work concerns the computational advantages that can be given by the use of a parallel computing scheme to carry out runs of the expensive objective function (Sóbester et al., 2004). If $N_{p}$ is the maximum number of possible parallel computations, whatever the local or global updating strategy, it is convenient to consider, at every update of the surrogate, the addition of a number of sampling points equal to $N_{p}$. These points could be taken as the $N_{p}$ optima belonging to the latest Pareto set, or the $N_{p}$ points which correspond to local maxima 
of the expected improvement function (the expected improvement function is generally multimodal). In alternative, some points could be taken among the Pareto set, some based on the expected improvement. This implies the definition of strategies that allow to identify and choose the aforementioned points (for example, a genetic algorithm with niching could be used to identify the multiple local minima, or a clustering procedure to identify the most representative designs within the Pareto set).

\subsubsection{Proposed updating strategy}

This section explains the strategy followed in this paper to identify the $N_{p}$ additional points at every surrogate update. The first step (Step 1) consists in choosing a number $p<N_{p}$ of points among the points belonging to the latest Pareto front. The choice of the points of the Pareto front may be carried out through clustering procedures; however in the following application, characterized by a low dimensionality of the objective function and design variable vectors, these points will be simply hand-picked. The second step (Step 2) consists in $q=N_{p}-p$ substeps (Step 2-1, . . Step 2-q): in each substep one of the remaining $q$ points is selected, based on a criterion which takes into account the expected improvement as well as the distance to the points already sampled or already selected. This criterion favors the addition of points where the expected improvement is higher, while at the same time avoiding that points are added close to locations already sampled or already selected.

At Step 2- $j$, the choice of a point is obtained by minimizing a function $F(\mathbf{x})$ which is a weighted sum of two metrics related to the two aspects (expected improvement and distance from the other points). The function 
$F(\mathbf{x})$ is defined as follows:

$$
F(\mathbf{x})=w_{c} \frac{c(\mathbf{x})}{c^{*}}+w_{E I} \frac{N L E I(\mathbf{x})}{N L E I^{*}}
$$

322

where $c$ is the metric related to the distance to the neighboring points, $c^{*}$ is a normalizing value, $N L E I$ is the metric related to the expected improvement and $N L E I^{*}$ its normalizing value, while $w_{c}$ and $w_{E I}\left(\right.$ with $\left.w_{c}+w_{E I}=1\right)$ are the weights.

To define the function $c(\mathbf{x})$, let us indicate with $\mathcal{Z}$ the set of the $z$ points already sampled (belonging to the sampling plan) or already selected (in Step 1 or in the preceding substeps of Step 2). Considering a candidate location $\mathbf{x}, \mathcal{Z}_{\mathbf{x}}$ is the subset of $\mathcal{Z}$ collecting the points which are neighbors of $\mathbf{x}$. One way to identify these neighbors is to consider the points, other than $\mathbf{x}$, representing vertices of Delaunay triangles which have $\mathbf{x}$ as one of the vertices.

The metric $c$ is defined as:

$$
c(\mathbf{x})=\frac{1}{z_{\mathbf{x}}} \sum_{x^{i} \in \mathcal{Z}_{\mathbf{x}}} \frac{1}{d\left(\mathbf{x}^{i}, \mathbf{x}\right)^{2}}
$$

where $z_{\mathbf{x}}$ is the number of points in $\mathcal{Z}_{\mathbf{x}}$, while $d\left(\mathbf{x}^{i}, \mathbf{x}\right)$ indicates the Euclidean norm:

$$
d\left(\mathbf{x}^{i}, \mathbf{x}\right)=\left[\sum_{j=1}^{n}\left|x_{j}^{i}-x_{j}\right|^{2}\right]^{1 / 2}
$$

$c(\mathbf{x})$ is therefore the average of a measure related to the inverse of the distances between point $\mathbf{x}$ and all the neighboring points in $\mathcal{Z}_{\mathbf{x}}$; in other words, the lower is $c$, the higher is the distance of $\mathbf{x}$ from its neighbors. The normalizing value $c^{*}$ is obtained by averaging the values of $c$ calculated for all 
341

the $h$ points belonging to the sampling plan:

$$
c^{*}=\frac{1}{h} \sum_{i=1}^{h} c\left(\overline{\mathbf{x}}^{i}\right)
$$

The function $N L E I(\mathbf{x})$ is defined as the negative of the logarithm of the expected improvement:

$$
N L E I(\mathbf{x})=-\log [E[I(\mathbf{x})]]
$$

therefore the lower is NLEI, the higher is the expected improvement. Having defined $\mathbf{x}^{b}$ as the point which minimizes $E[I]$, the normalizing value of $N L E I$ is defined as:

$$
N L E I^{*}=N L E I\left(\mathbf{x}^{b}\right)=-\log \left[E\left[I\left(\mathbf{x}^{b}\right)\right]\right]
$$

The updating procedure can be terminated when a user-defined convergence criterion is met.

\subsection{Flow simulation}

The CFD investigation of flow around a civil structure immersed in the atmospheric boundary layer is extremely challenging due to the flow complexity and the associated computational cost. Generally speaking, for an unsteady incompressible flow simulation, having chosen an appropriate discretization scheme and a grid that can be considered fine enough, the quality of the simulation results will depend mainly on the turbulence modeling. The choice of the most appropriate turbulence modeling scheme will be guided by two generally conflicting aspects: on the one hand, the accuracy of the results that the scheme is able to deliver, considering many factors such as the type of geometry, the Reynolds number range, the type of features of the 
flow that need to be captured by the simulation, the bidimensional or tridimensional nature of the problem; and on the other hand, the computational weight of the analyses. In this work, URANS (unsteady Reynolds-averaged Navier-Stokes) simulations are considered capable of describing the features of the flow around the bluff body of interest. While other kinds of simulation methods (e.g. large-eddy simulation or hybrid methods such as detached eddy simulation (Spalart et al., 2004) or delayed detached-eddy simulation (Spalart et al., 2006)) could give more accurate results, URANS is considered a good trade-off between the efficacy in representing the features of interest of the flow and the computational time necessary for carrying out the analyses. While the accuracy of the analyses is obviously important to the reliability of the results, a discussion on this topic is outside the scope of this paper; it should be observed that the real challenge - and the practical purpose of the use of CFD in ASO is to find a shape which performs better than the original shape (Jameson, 2004). Also, the quality of the simulations does not affect the general validity of the method presented.

As a means of expediting the preprocessing phase of the CFD analyses, it is here proposed the adoption of a mesh morphing algorithm (Wei et al., 2014), that allows the automatic generation of the meshes for all the geometries on which CFD analyses are run, commencing from a manually defined mesh for a baseline geometry. As an additional advantage, this also ensures the consistency of all the meshes used in the calibration of the surrogate model. 
400

\section{Case study}

\subsection{Description}

As a first application of the shape optimization framework proposed in this paper, the optimization of a bi-dimensional shape, representing the crosssection of a tall building, is considered. As shown in Fig. 1, the baseline shape is a square of unitary width with rounded corners with curvature radius of 0.4. Due to the presence of four axes of symmetry, the geometry is fully described by one eighth of the shape. The straight segments of the shape's perimeter are fixed, while the shape of the rounded parts can change, according to the positions of two control points. The two design variables represent the displacements of the control points, with respect to the position in the baseline configuration, along the direction $y^{*}$, and are indicated as $\Delta y_{1}^{*}$ and $\Delta y_{2}^{*}$. The resulting shape of the corner is obtained through interpolation with a cubic spline of the positions of the control points, with tangent parallel to the straight segment at the connection with this last.

[Figure 1 about here.]

The mathematical formulation of the optimization problem for the case study is as follows:

$$
\begin{aligned}
& \text { Find } \mathbf{q}=\left(\Delta y_{1}^{*}, \Delta y_{2}^{*}\right) \\
& \text { to minimize } \mathbf{G}(\mathbf{q})=\left(\mu_{C_{d}}(\mathbf{q}), \sigma_{C_{l}}(\mathbf{q})\right)
\end{aligned}
$$

subject to:

$$
\Delta y_{\min } \leq \Delta y_{i}^{*} \leq \Delta y_{\max } \quad i=1,2
$$

where $\mu_{C_{d}}$ indicates the mean value of the drag coefficient, $\sigma_{C_{l}}$ the standard deviation of the lift coefficient while $\Delta y_{\min }=-0.1$ and $\Delta y_{\max }=0.1$. 
A maximum of number $N_{p}=15$ parallel computations were available, with a total budget of 90 analyses. Therefore, the initial sampling plan (determined, as anticipated, through Latin hypercube sampling with MorrisMitchell criterion) comprised 15 points and, at each of the successive updating cycles, 15 points were added, for a maximum of 5 updating cycles. The updates were carried out through the procedure outlined in Section 3.3.2, with $w_{c}=0.2$ and $w_{E I}=0.8$ (Eq. 15). The sampling plan is shown in Fig. 2 .

[Figure 2 about here.]

As previously discussed, URANS simulations have been adopted for running the analyses on the sampling points. For each simulation, meshes were obtained through the mesh morphing algorithm illustrated in Wei et al. (2014) commencing from a multi-block structured mesh for the baseline geometry (see Fig. 3).

The $k-\omega$ SST (Shear Stress Transport) formulation (Menter, 1992) was used to model the Reynolds stress and was implemented in the OpenFOAM framework (Jasak et al., 1999), an open-source CFD code that uses a collocated finite volume method on arbitrary unstructured grids (http://www . openfoam.com/). The PISO algorithm (Issa, 1986) was used to solve the coupled velocity pressure equations. The SST model was used in Low-Reynolds-Number mode, that is no wall function is employed, the mesh is given a fine enough resolution to allow the flow to be resolved down to the wall $\left(y_{1 s t}^{+}<1\right)$, while the wall grid stretching ratio is lower than 1.1. Figure 4 shows snapshots of the velocity fields obtained through the simulations for some configurations of the sampling plan and for the baseline configuration. As can be seen, signif- 
icant changes in the flow field around the cross-section can be achieved by varying the geometry of the cross-section within the prescribed limits. This should ensure that the design space of the case study will be characterized by objective functions that can vary over a relatively large interval, therefore defining a minimization problem of practical interest.

[Figure 3 about here.]

[Figure 4 about here.]

The two independent kriging surrogates used for approximating the expensive objective functions are indicated as $\hat{\mathbf{G}}(\mathbf{q})=\left(\hat{\mu}_{C_{d}}(\mathbf{q}), \hat{\sigma}_{C_{l}}(\mathbf{q})\right)$. The sample values of $\mu_{C_{d}}$ and $\sigma_{C_{l}}$ are obtained from the flow field when it becomes statically stationary. In particular, $\mu_{C_{d}}$ and $\sigma_{C_{l}}$ are averaged over at least 10 vortex shedding cycles. Figures 5(a) and 5(b) show the two kriging surfaces initially obtained through fitting to the set of 15 points of the sampling plan.

[Figure 5 about here.]

The optimization algorithm adopted for the solution of the problem outlined in Eqs. 21 to 23 is the well-known NSGA-II, proposed by Deb et al. (2002). The population size was set to 20, with 2 elite individuals and $80 \%$ of the remaining individuals found through cross-over. The analyses were terminated after 400 generations.

\subsection{Results and discussion}

Figure 6(a) shows the Pareto front obtained after an optimization run considering the two initial kriging models of Figs. 5(a) and 5(b). To evaluate the validity of this initial front as well as the overall predictive capability 
of the kriging models, the validation and improvement strategy of Section 3.3.2 was invoked. Figure 6(a) shows 15 added points obtained applying the aforementioned scheme. In particular, in Step $1 p=4$ points were chosen from the initial Pareto front while the remaining 11 points were assigned following Step 2. The location of the points within the space of the design variables is shown in Fig. 7(a) together with the contour of the expected improvement. In order to define a measure of the overall capability of the kriging models to estimate the objective functions, the distance between the value predicted by the kriging model and that found by directly evaluating the objective functions is considered. Figure 6(a) graphically shows this distance for the 15 points of the validation and improvement scheme. As can be seen from Fig. 6(a), there exists a certain discrepancy between the values predicted by the kriging models and the actual values assumed by the objective functions. By re-calibrating the kriging models to the initial 15 points as well as those identified through the updating strategy, a new optimization run can be carried out with the identification of a new Pareto front. This front is shown in Fig. 6(b) together with the 15 points once again identified through the proposed updating strategy and shown in Fig. 7(b). In particular, 7 points were chosen from the estimated Pareto front (Step 1) while the remaining 8 were chosen following Step 2. As is intuitively visible from Fig. 6(b), the predicted and the actual objective function values still show a certain inconsistency. This is better illustrated in Fig. 8 where the maximum and average distances are reported for each updating cycle of this case study. In particular, it can be seen that the aforementioned distances grow in the second cycle. By repeating this updating strategy (with 
7 points picked on the front for update 3 and 8 points for update 4), the fronts shown in Figs. 6(c) to 6(e) are found. The location, in the design variable space, of these added points are shown in Figs. 7(c) and 7(d). The change in the predictive capability of the kriging model as the points are added during the design updates is shown in Fig. 8. In particular, it can be seen that for the last three designs it remains relatively constant, where for the validation of the last update (shown in Fig. 8) 11 points were chosen from the Pareto front shown in Fig. 6(e) (Step 1), while 4 according to Step 2 (Fig. 7(e)). The stability of the Pareto front found in this last update is once again investigated by comparing the kriging predictions to actual function values, as shown in Fig. 6(e) and in more detail in Fig. 6(f). While there is still a discrepancy between the kriging predictions and the actual objective functions, the location within the space of the design variables of the Pareto optimal solutions would seem to have been identified. To better investigate this, it is interesting to consider the subset of directly evaluated points within the space of the objective functions that constitute an estimate of the Pareto front. These points are shown in Fig. 9(b) together with the complete set of points evaluated during the proposed surrogate-based optimization strategy. Figure 9 (a) also shows the location of the front within the geometric space of the design variables. From Fig. 9(a), it is evident that the Pareto optimal designs have similar geometric configurations. This is further illustrated in Fig. 10(a) where the geometries of three representative points of the estimated Pareto front are shown. This figure also illustrates how the optimal geometries are represented by a typical square with double recessed corners. This result would seem to suggest the validity of the pro- 
posed approach as this configuration is known to be optimum for a cylinder in turbulent flow. Figures 9(a) and 9(b) also show the location and aerodynamic performance of the baseline configuration. It is interesting to observe the evident aerodynamic advantages compared to this baseline gained from applying the proposed optimization strategy. Returning to the kriging estimated Pareto front of Fig. 6(f), it is interesting to observe that, although there is some discrepancy between the predicted and actual values of the objective functions, the geometries corresponding to the predicted front are extremely similar to those of the front estimated directly from the configurations evaluated during the optimization process. This is shown in Fig. 10(b) where the geometries of the end points as well as central point of the kriging-estimated Pareto front are shown. The close correspondence between the geometries of Figs. 10(a) and 10(b) clearly illustrate the potential of the proposed ASO approach. Finally, it should be observed that, in order to find the Pareto fronts discussed here, a total of 90 CFD simulations were carried out while a total of about 12000 evaluations of the kriging models were made at each design update. Therefore, by following the proposed approach, only $0.75 \%$ of the CFD runs necessary to directly search for the Pareto optimal solution are necessary, which once again illustrates the strong potential of the proposed ASO approach.

[Figure 6 about here.]

[Figure 7 about here.]

[Figure 8 about here.]

[Figure 9 about here.] 
[Figure 10 about here.]

\section{Conclusions and future directions}

This paper reported an innovative aerodynamic shape optimization strategy for the identification of optimal shapes of civil structures immersed in turbulent flow. In particular, a multiobjective surrogate-based approach was proposed in which ordinary kriging was used not only to build the surrogates of the aerodynamic measures to be controlled, but also for defining a novel validation and updating scheme. The applicability of the proposed strategy was investigated on a case study represented by the cross-section of a cylindrical tall building. The goal was to minimize the mean drag coefficient as well as the standard deviation of the lift coefficient while modeling the turbulent flow through unsteady Reynolds-averaged Navier-Stokes simulations. The results of this case study clearly illustrated the potential of the approach. Indeed, in order to find an estimate of the Pareto front of the problem, only $0.75 \%$ of the CFD simulations that would have been necessary to directly estimate the front were needed. Having said this, a certain discrepancy between the aerodynamic measures predicted by the ordinary kriging models and the actual values assumed by the measures was observed. While this did not seem to hinder the capability of the proposed approach for identifying the aerodynamically optimum solutions, it is an area which deserves further investigation. In particular, the possibility of using a different kind of surrogate, as for example regression-kriging or support vector regression, as well as a two-step approach where a new surrogate-based optimization is run in a subset of the original design space will be investigated in 
future work. In conclusion, this paper would seem to suggest the strong potential of surrogate-based multiobjective optimization schemes for obtaining aerodynamic configurations of civil structures that effectively reduce their aerodynamic impact.

\section{Acknowledgments}

Support for this work was provided by the NSF Grant No. CMMI1301008.

\section{References}

Abdelrazaq, A., Kijewski-Correa, T., Song, Y.-H., Case, P., Isyumov, N., Kareem, A., 2004. Design and full-scale monitoring of the tallest building in Korea: Tower Palace III. In: 6th Asian-Pacific Conference on Wind Engineering.

Ahmed, M., Qin, N., 2009. Surrogate-based aerodynamic design optimization: Use of surrogates in aerodynamic design optimization. In: 13th International Conference on Aerospace Science and Aviation Technology.

Arias-Montaño, A., Coello Coello, C. A., Mezura-Montes, E., 2012. Multiobjective evolutionary algorithms in aeronautical and aerospace engineering. Evolutionary Computation, IEEE Transactions on 16 (5), 662-694.

Baker, W., Korista, D., Novak, L., 2007. Burj Dubai: engineering the world's tallest building. The Structural Design of Tall and Special Buildings 16, $361-375$. 
Deb, K., 2001. Multi-objective optimization using evolutionary algorithms. Vol. 16. Wiley.

Deb, K., Pratap, A., Agarwal, S., Meyarivan, T., 2002. A fast and elitist multiobjective genetic algorithm: NSGA-II. Evolutionary Computation, IEEE Transactions on 6 (2), 182-197.

Forrester, A. I. J., Sóbester, A., Keane, A. J., 2008. Engineering design via surrogate modelling. A practical guide. John Wiley and Sons, Ltd.

Issa, R. I., 1986. Solution of the implicitly discretised fluid flow equations by operator-splitting. Journal of Computational Physics 62, 40-65.

Jameson, A., 2004. Efficient aerodynamic shape optimization. In: 10th AIAA/ISSMO Multidisciplinary Analysis and Optimization Conference.

Jasak, H., Weller, H. G., Gosman, A. D., 1999. High resolution NVD differencing scheme for arbitrarily unstructured meshes. International Journal for Numerical Methods in Fluids 31 (2), 431-449.

Jeong, S., Minemura, Y., Obayashi, S., 2006. Optimization of combustion chamber for diesel engine using kriging model. Journal of Fluid Science and Technology 1, 138-146.

Jones, D. R., 2001. A taxonomy of global optimization methods based on response surfaces. Journal of Global Optimization 21, 345-383.

Jones, D. R., Schonlau, M., Welch, W. J., 1998. Efficient global optimization of expensive black-box functions. Journal of Global Optimization 13, 455492. 
Kareem, A., Bernardini, E., Spence, S. M. J., 2013a. Control of the wind induced response of structures. Springer Japan, Ch. 14, pp. 377-410.

Kareem, A., Bobby, S., Spence, S. M. J., Bernardini, E., 2014. Optimizing the form of tall buildings to urban environments. In: CTBUH 2014 International Conference.

Kareem, A., Kijewski, T., Tamura, Y., 1999. Mitigation of motions of tall buildings with specific examples of recent applications. Wind and Structures 3 (2), 201-251.

Kareem, A., Spence, S. M. J., Bernardini, E., Bobby, S., Wei, D., 2013b. Using computational fluid dynamics to optimize tall building design. CTBUH Journal (III), 38-42.

Keane, A. J., 2006. Statistical improvement criteria for use in multiobjective design optimization. AIAA Journal 44, 879-891.

Kim, Y., Kanda, J., Tamura, Y., 2011. Wind-induced coupled motion of tall buildings with varying square plan with height. Journal of Wind Engineering and Industrial Aerodynamics 99 (5), 638-650.

Kleijnen, J. P. C., 2009. Kriging metamodeling in simulation: A review. European Journal of Operational Research 192, 707-716.

Mackay, M. D., Beckman, R. J., Conover, W. J., 1979. A comparison of three methods for selecting values of input variables in the analysis of output from a computer code. Technometrics 21, 239-245. 
Madsen, J., Shyy, W., Haftka, R., 2000. Response surface techniques for diffuser shape optimization. AIAA Journal 38, 1512-1518.

Menter, F., 1992. Performance of popular turbulence model for attached and separated adverse pressure gradient flows. AIAA Journal 30, 2066-2072.

Mohammadi, G., Pironneau, O., 2001. Applied shape optimization for fluids. Oxford University Press.

Morris, M. D., Mitchell, T. J., 1995. Exploratory designs for computer experiments. Journal of Statistical Planning and Inference 43, 381-402.

Queipo, N. V., Haftka, R., Shyy, W., Goel, T., Vaidyanathan, R., Tucker, P. K., 2005. Surrogate-based analysis and optimization. Progress in Aerospace Sciences 41, 1-28.

Sacks, J., Welch, W. J., Mitchell, T. J., Wynn, H. P., 1989. Design and analysis of computer experiments. Statistical Sciences 4, 409-423.

Schonlau, M., Welch, W. J., Jones, D. R., 1998. Global versus local search in constrained optimization of computer models. In: New Developments and Applications in Experimental Design. Vol. 34 of Lecture Notes - Monograph Series. Institute of Mathematical Statistics, pp. 11-25.

URL http://www.jstor.org/stable/4356058

Shimoyama, K., Jeong, S., Obayashi, S., 2013. Kriging-surrogate-based optimization considering expected hypervolume improvement in nonconstrained many-objective test problems. In: IEEE Congress on Evolutionary Computation. pp. 658-665. 
Sóbester, A., Leary, S., Keane, A., 2004. A parallel updating scheme for approximating and optimizing high fidelity computer simulations. Structural and Multidisciplinary Optimization 27 (5), 371-383.

URL http://dx.doi .org/10.1007/s00158-004-0397-9

Spalart, P. R., Deck, S., Shur, M. L., Squires, K. D., Strelets, M. K., Travin, A., 2006. A new version of detached-eddy simulation, resistant to ambiguous grid densities. Theoretical and Computational Fluid Dynamics 20, 181-195.

Spalart, P. R., Jou, W.-H., Strelets, M., Allmaras, S. R., 2004. Comments on the feasibility of LES for wings, and on a hybrid RANS/LES approach. In: First AFOSR International Conference on DNS/LES.

Spence, S. M. J., Bernardini, E., Wei, D., Kareem, A., Wu, T., 2013. A lowdimensional model for the aerodynamic shape optimization of tall buildings. In: 12th Americas Conference on Wind Engineering.

Tanaka, H., Tamura, Y., Ohtake, K., Nakai, M., Kim, Y., 2012. Experimental investigation of aerodynamic forces and wind pressures acting on tall buildings with various unconventional configurations. Journal of Wind Engineering and Industrial Aerodynamics 107-108, 179-191.

Thévenin, D., Janiga, G. (Eds.), 2008. Optimization and Computational Fluid Dynamics. Springer-Verlag.

Wei, D., Spence, S. M. J., Kareem, A., Jemcov, A., 2014. A structured mesh boundary motion approach for simulating wind effects on bluff bodies 
658 with changing boundaries. Journal of Wind Engineering and Industrial 659 Aerodynamics 126, 118-131.

660 Xie, J., 2014. Aerodynamic optimization of super-tall buildings and its effec661 tiveness assessment. Journal of Wind Engineering and Industrial Aerody662 namics $130,88-98$. 


\section{List of Figures}

1 Reference geometry of the case study. The two control points are placed at $x^{*}=-0.16$ (control point 1 ) and $x^{*}=-0.04$ (control point 2). . . . . . . . . . . . . . . . 32

2 Sampling plan: (a) the 15 configurations in the design variable space; (b) configurations and baseline geometry (one quarter of the geometry shown). . . . . . . . . . . . . . . . . 33

3 Meshes for some of the configurations: (a) baseline, (b) configuration 1, (c) configuration 14 and (d) configuration 15. . . 34

4 Snapshots of the velocity field obtained from URANS simulation for some of the configurations: (a) baseline, (b) configuration 1, (c) configuration 14 and (d) configuration 15. . . . . 35

5 Kriging surrogates: models for (a) $\mu_{C_{d}}$ and (b) $\sigma_{C_{l}}$ fitted to the sampling plan. . . . . . . . . . . . . . . . . 36

6 Space of the objective functions, predictive capabilities of the kriging models for (a) first kriging (fitted to the sampling plan), (b) second (fitted to 30 points), (c) third (fitted to 45 points), (d) fourth (fitted to 60 points), (e)-(f) fifth (fitted to 75 points). The distance between the points sampled for validation and their kriging prediction is highlighted. The predicted Pareto front is also shown. . . . . . . . . . . . . . 37

7 Sampled points, contours of the expected improvement and points added at each updating/validation cycle: (a) first, (b) second, (c) third, (d) fourth and (e) fifth updating/validation cycle. . . . . . . . . . . . . . . . 38

8 Distances between the kriging-based estimates and the CFDbased evaluations. At each updating cycle, the average and maximum distance, in the objective function space, observed for the set of 15 points is shown; the curves are normalized at the values assumed at the first updating cycle. . . . . . . . . . 39

9 Pareto front estimated directly from the 90 sampled points: (a) close-up of design variable space; (b) objective function space. . . . . . . . . . . . . . . . . . 40

10 Optimal geometric configurations: (a) three optimal geometries belonging to the Pareto front shown in Fig. 9; (b) three optimal geometries belonging to the Pareto front found after the fourth updating cycle shown in Fig. 6(f). . . . . . . . . . . 41 


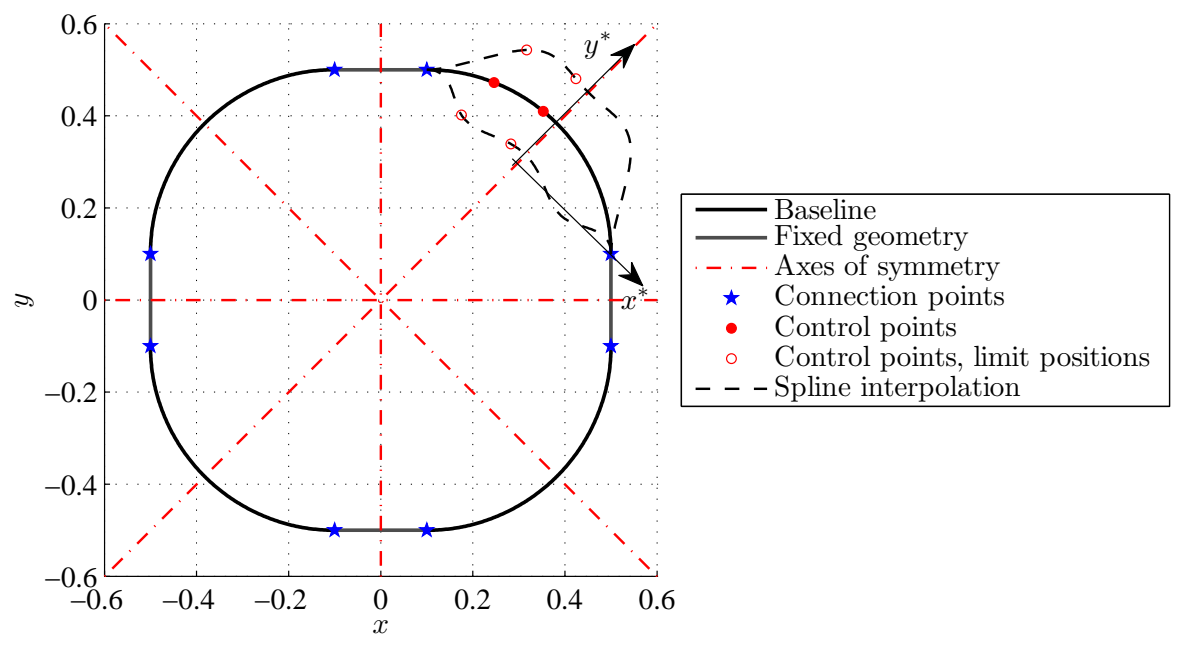

Figure 1: Reference geometry of the case study. The two control points are placed at $x^{*}=-0.16$ (control point 1$)$ and $x^{*}=-0.04$ (control point 2 ). 


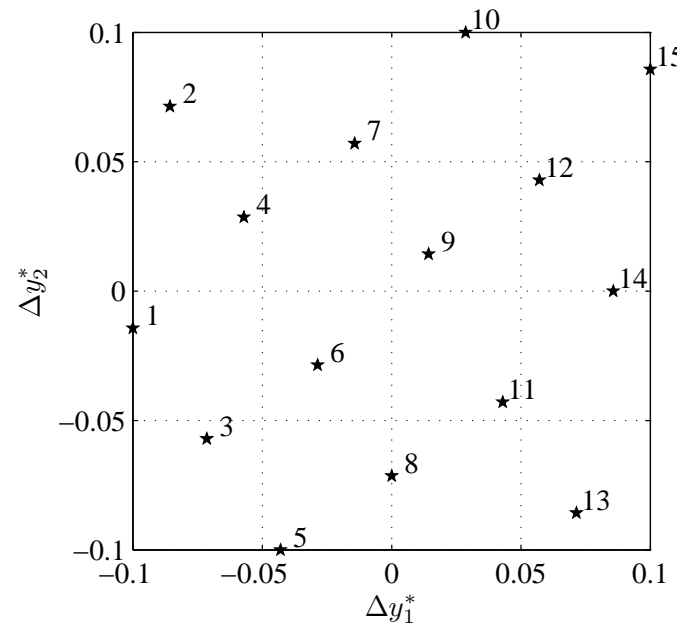

(a)

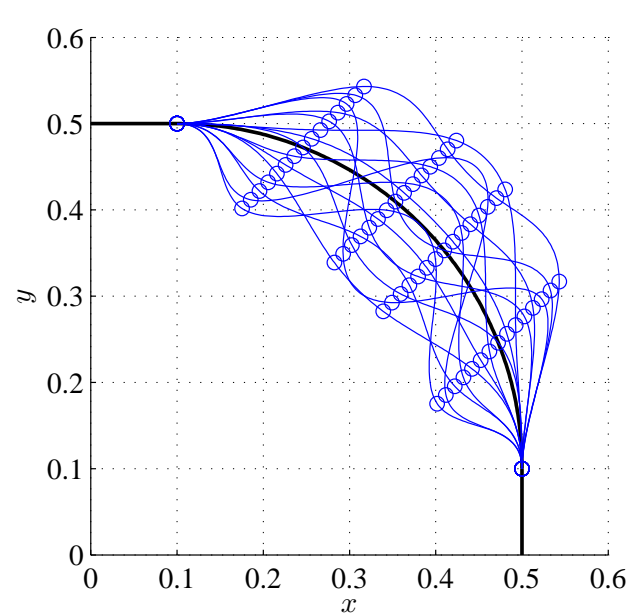

(b)

Figure 2: Sampling plan: (a) the 15 configurations in the design variable space; (b) configurations and baseline geometry (one quarter of the geometry shown). 


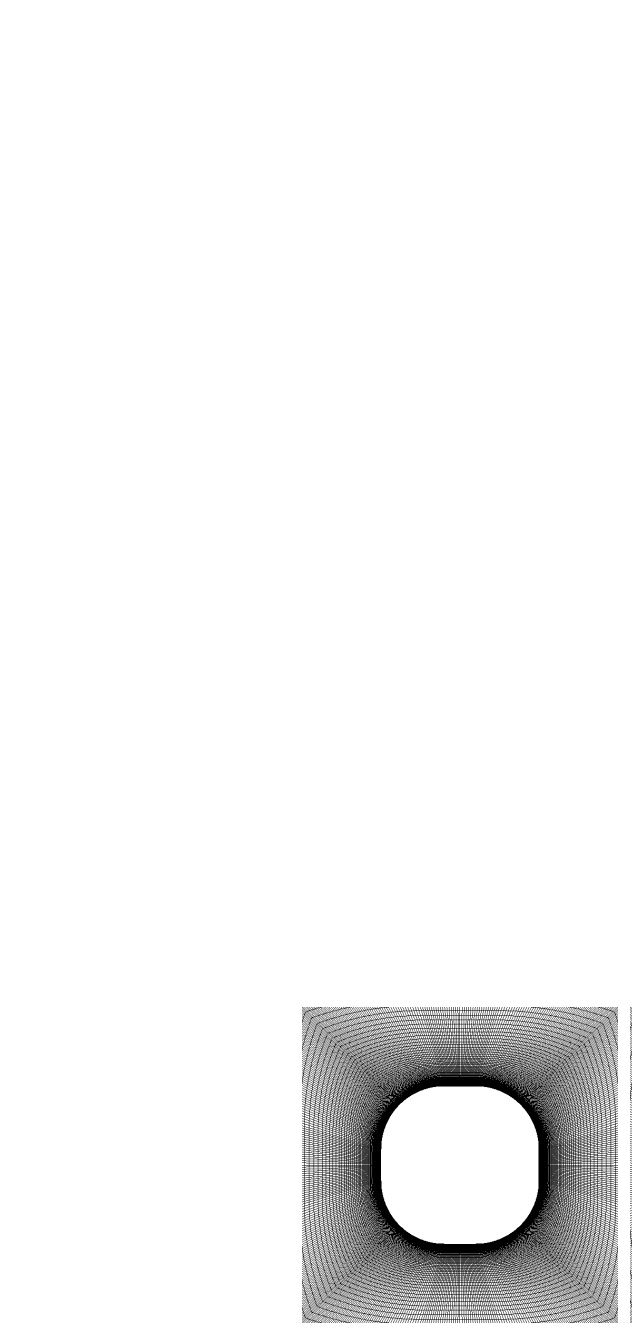

(a)

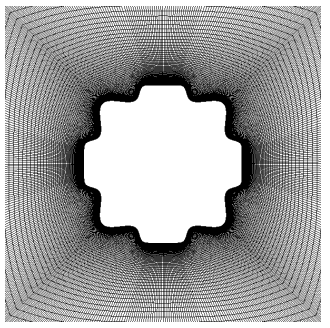

(b)

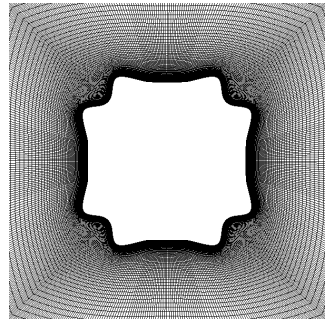

(c)

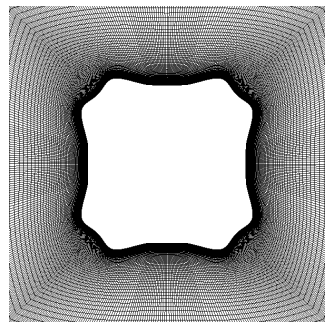

(d)

Figure 3: Meshes for some of the configurations: (a) baseline, (b) configuration 1, (c) configuration 14 and (d) configuration 15. 


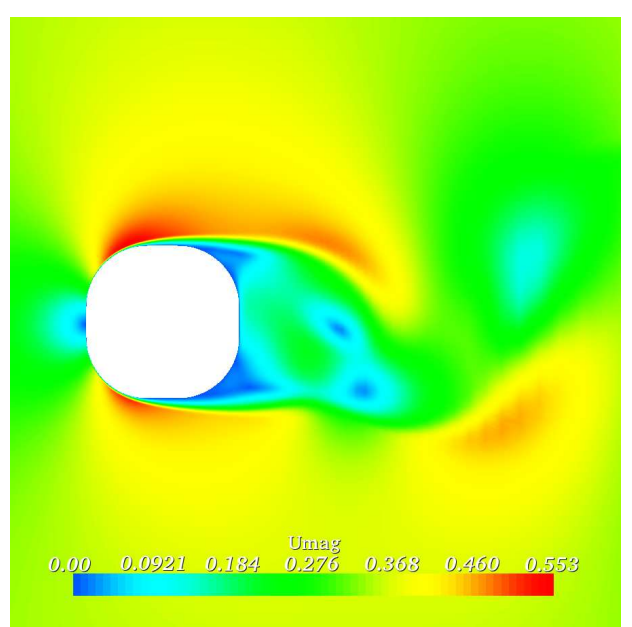

(a)

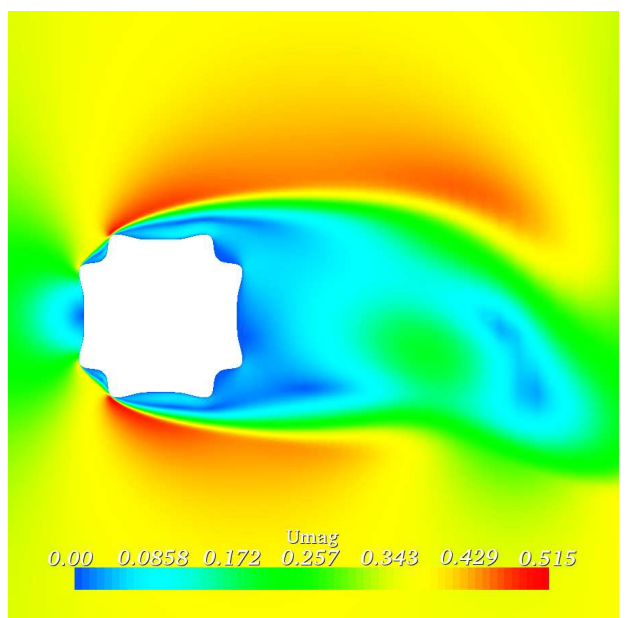

(c)

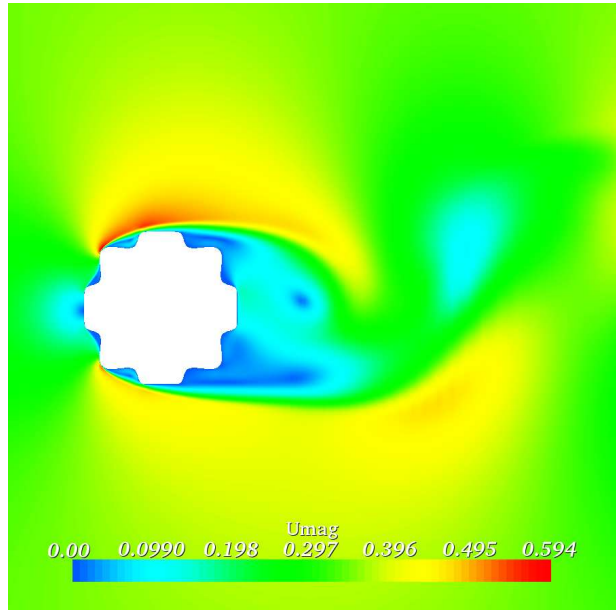

(b)

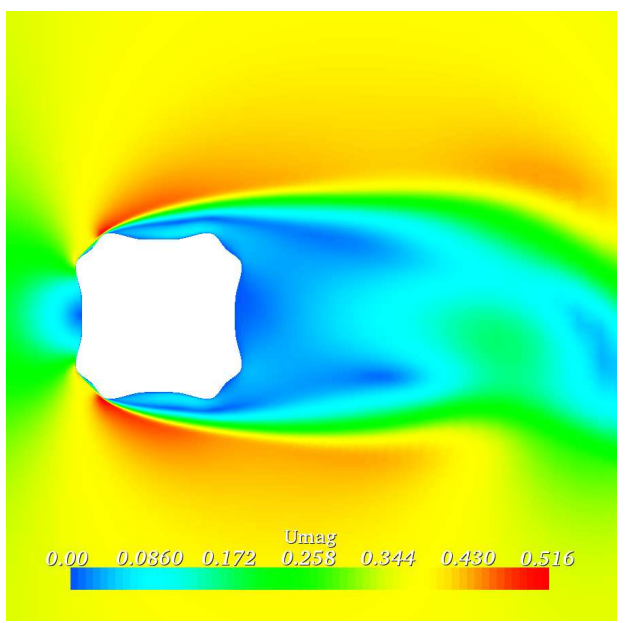

(d)

Figure 4: Snapshots of the velocity field obtained from URANS simulation for some of the configurations: (a) baseline, (b) configuration 1, (c) configuration 14 and (d) configuration 15 . 


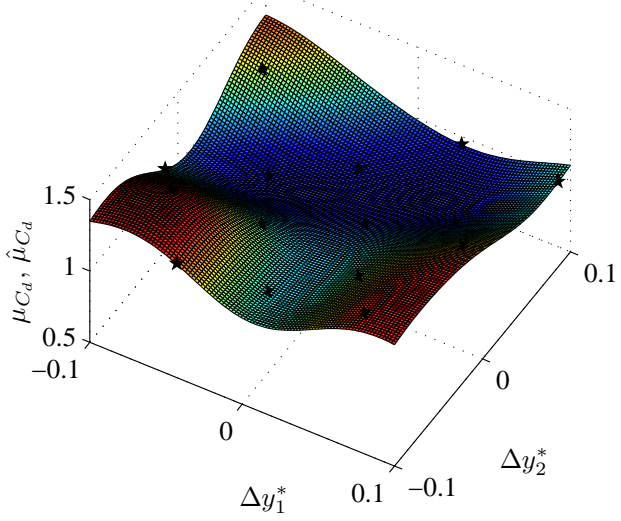

(a)

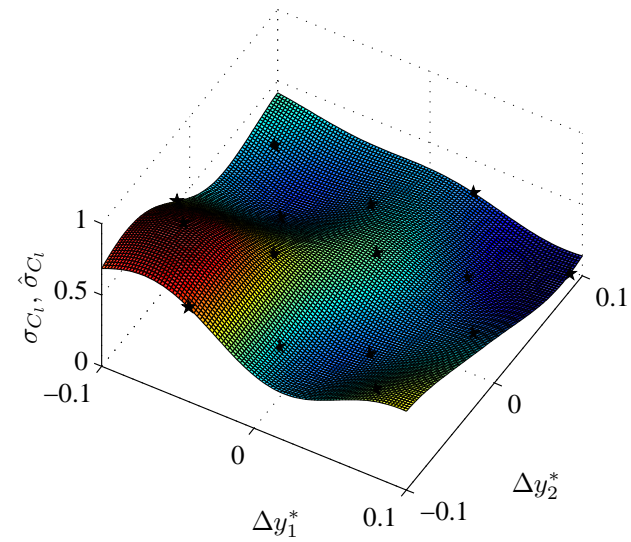

(b)

Figure 5: Kriging surrogates: models for (a) $\mu_{C_{d}}$ and (b) $\sigma_{C_{l}}$ fitted to the sampling plan. 


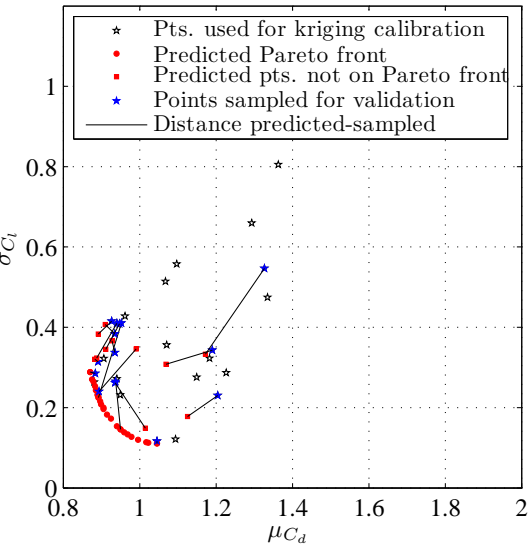

(a)

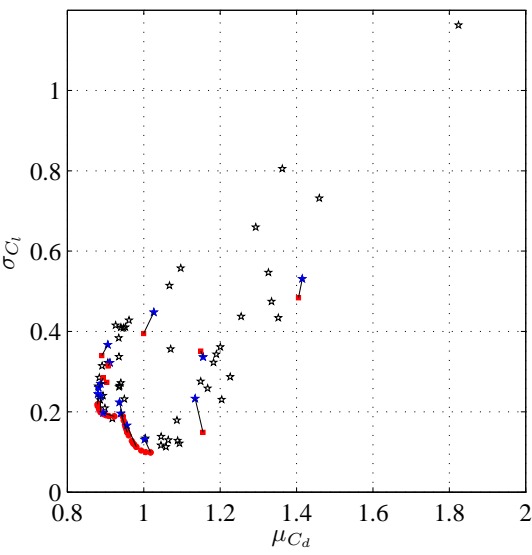

(c)

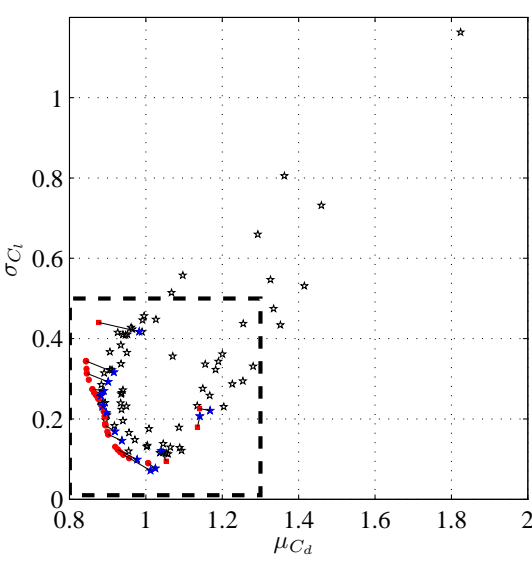

(e)

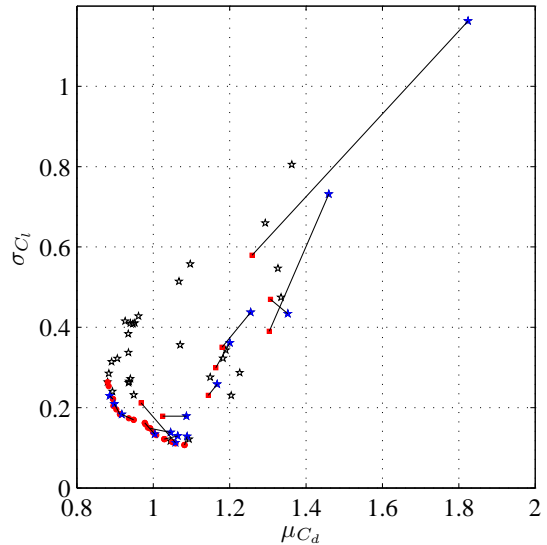

(b)

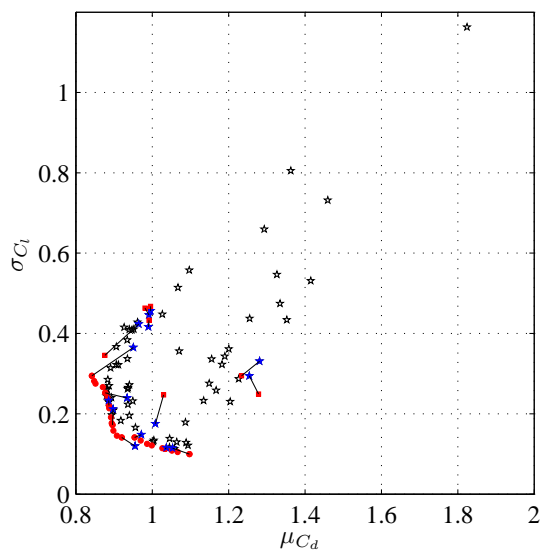

(d)

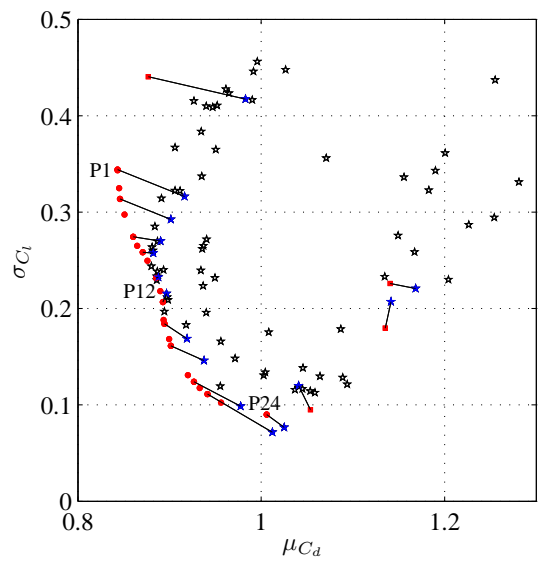

(f)

Figure 6: Space of the objective functions, p88dictive capabilities of the kriging models for (a) first kriging (fitted to the sampling plan), (b) second (fitted to 30 points), (c) third (fitted to 45 points), (d) fourth (fitted to 60 points), (e)-(f) fifth (fitted to 75 points). The distance between the points sampled for validation and their kriging prediction is highlighted. The predicted Pareto front is also shown. 


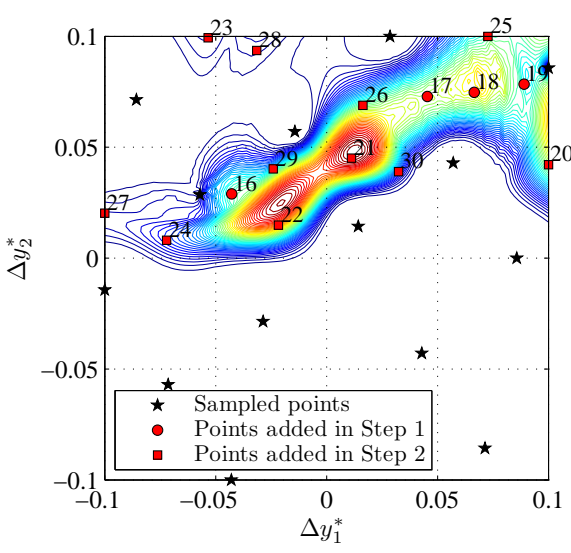

(a)

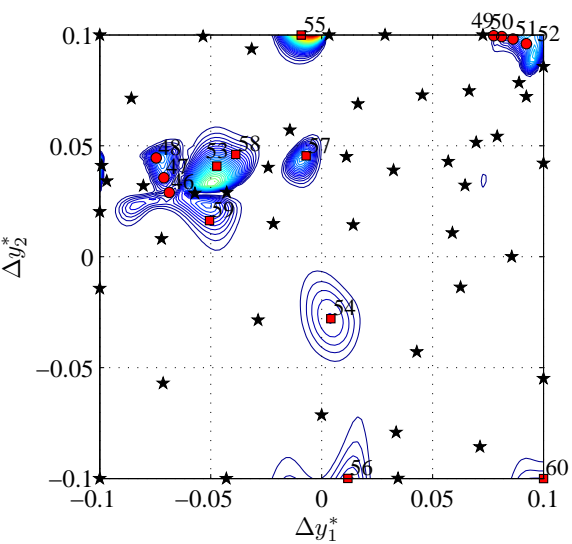

(c)

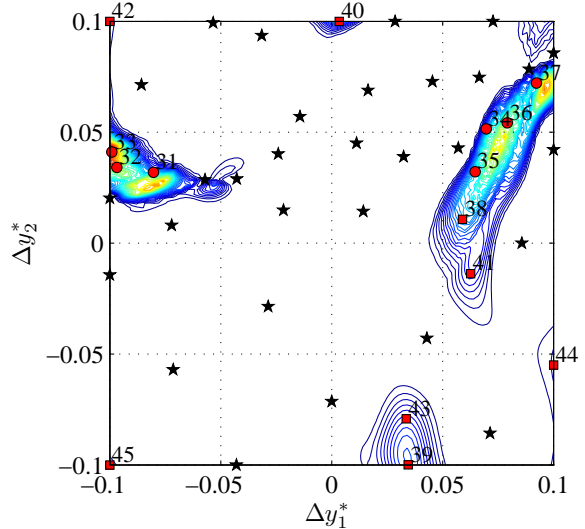

(b)

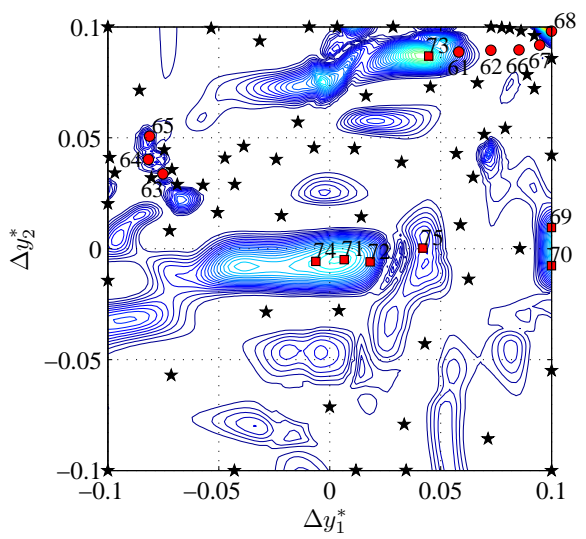

(d) 


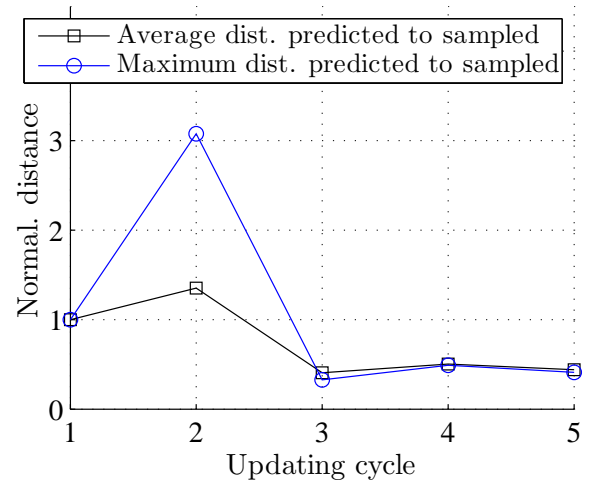

Figure 8: Distances between the kriging-based estimates and the CFD-based evaluations. At each updating cycle, the average and maximum distance, in the objective function space, observed for the set of 15 points is shown; the curves are normalized at the values assumed at the first updating cycle. 


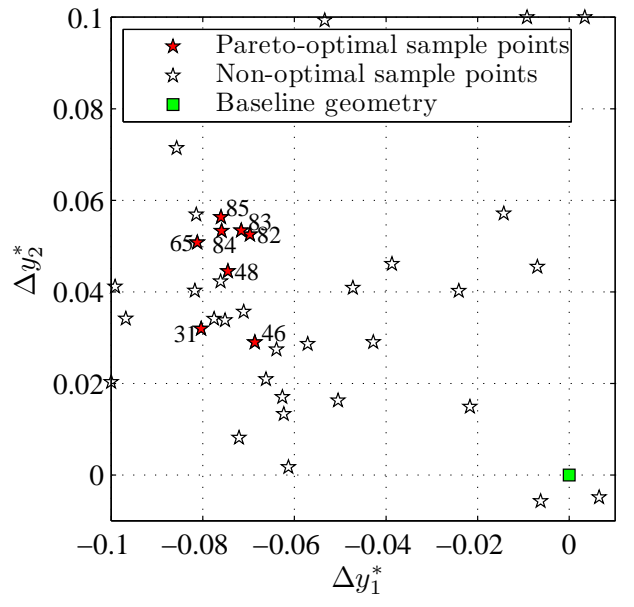

(a)

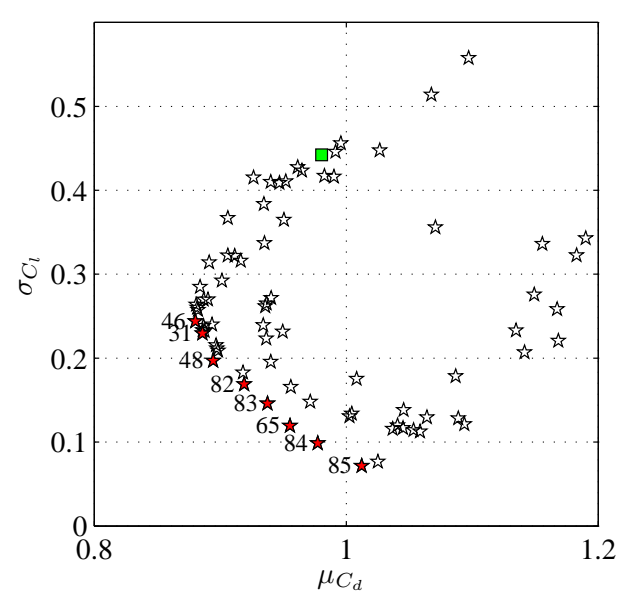

(b)

Figure 9: Pareto front estimated directly from the 90 sampled points: (a) close-up of design variable space; (b) objective function space. 


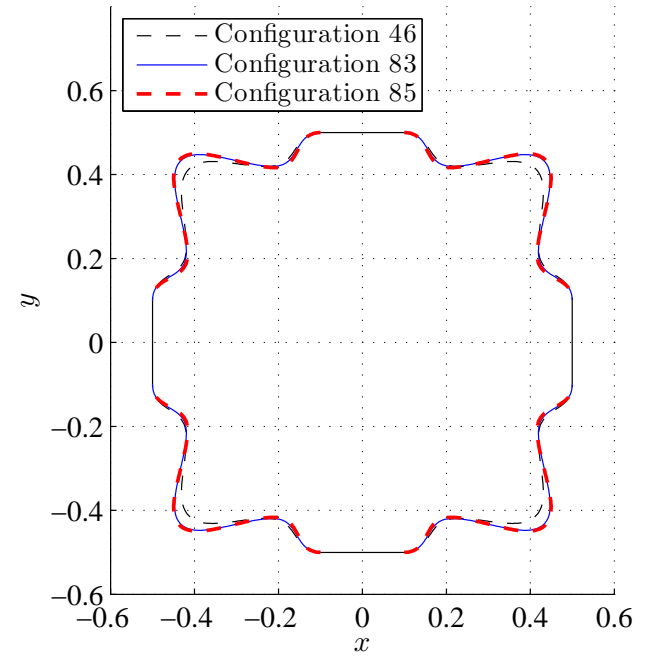

(a)

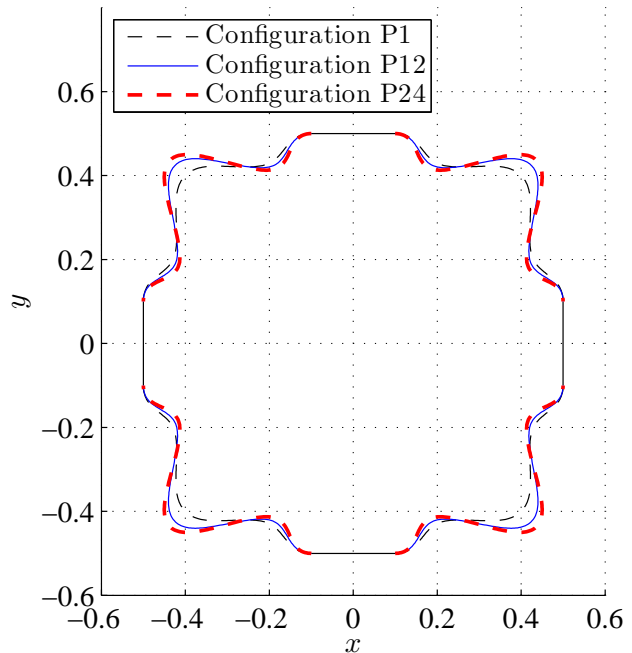

(b)

Figure 10: Optimal geometric configurations: (a) three optimal geometries belonging to the Pareto front shown in Fig. 9; (b) three optimal geometries belonging to the Pareto front found after the fourth updating cycle shown in Fig. 6(f). 